\title{
Primary infradiafragmatic bony classical Hodgkin lymphoma
}

\author{
Fariba Binesh $^{1,2}$, Reza Nafisi Moghadam ${ }^{3}$, Mohammad Reza Vahidfar ${ }^{4}$, Ehsan Amiri , \\ Fatemeh Pourhosseini ${ }^{1}$, Seyed Hossein Shahcheraghi ${ }^{5 *}$
}

1 Department of Pathology, Shahid Sadoughi University of Medical Sciences, Yazd, Iran

2 Hematology and Oncology Research Center, Shahid Sadoughi University of Medical Sciences and Health Services, Yazd, Iran 3 Department of radiology, Shahid Sadoughi University of Medical Sciences, Yazd, Iran

4 Hematooncologist, Yazd, Iran

5 Infectious Diseases Research Center, Shahid Sadoughi Hospital, Shahid Sadoughi University of Medical Sciences, Yazd, Iran

*Corresponding Author: Seyed Hossein Shahcheraghi, Infectious Diseases Research Center, Shahid Sadoughi Hospital, Shahid Sadoughi University of Medical Sciences, Yazd, Iran

Email: shahcheraghih@gmail.com, Tel: 00989132531389

\section{Abstract}

Infradiaphragmatic Hodgkin's lymphoma (IDHL) is a scarce neoplasm and primary pelvic bone exhibition is limited to case reports. Recognition and management of likewise a rare disease are challenging. Here the authors present a peculiar case of classic Hodgkin lymphoma (CHL) of pelvic bone. A 31year-old man was admitted to our hospital with chief complaint of left hip pain. Imaging showed a lytic lesion in the left iliac bone with sclerotic margin and extension to soft tissue. Open biopsy confirmed the diagnosis of CHL. CHL at first introducing as pelvic bone involvement is really uncommon. The diagnosis of primary bone $\mathrm{CHL}$ should be made by tough histological and clinical expression.

Keywords: Classical Hodgkin lymphoma, Bony Hodgkin lymphoma, Infradiafragmatic.

\section{INTRODUCTION}

Hodgkin lymphoma includes a specific group of tumors that are defined by the attendance of a tumor giant cell, the ReedStenberg cells cell. In late stage HL, bone involvement has been detected in $10 \%$ to $20 \%$ of patients ${ }^{[1]}$. However, primary bony $\mathrm{HL}$ really exists and just very rare literatures presented [2-5]. Recognition of single bone involvement in HL cases as "primary" is difficult as the diagnosis of primary bony $\mathrm{HL}$ should be confirmed according to histological and clinical presentations. Indeed primary bony $\mathrm{HL}$ is an early stage disease, but secondary bone involvement demonstrates a progressive one. IDHL counts for $3-11 \%$ of adult patients of early stages of Hodgkin lymphoma ${ }^{[6]}$, here we present a 31year-old man with a left pelvic involvement which confirmed to be classical HL.

\section{CASE REPORT}

A 31year-old man was referred to our hospital with a chief complaint of 8 months of left hip pain. The patient reported a history of night sweats, low-grade fever, and approximately $7 \%$ weight loss during the past 6 months. Also, he discovered a lump in his left hip during the latter month. Physical examination suggested the compaction of sciatic nerve. Lab data revealed that the WBC (white blood cell) count was $15 \times 10^{9} \mathrm{~L}$ with the neutrophils rate of $60 \%$ and lymphocyte rate of $40 \%, \mathrm{Hb}$ was $95 \mathrm{~g} / \mathrm{L}$, platelet count was $250 \times 10^{9} \mathrm{~L}$. Epstein-Barr virus test was not done. Bone marrow aspiration showed a normocellular marrow with mild eosinophilia. A Coronal and axial pelvic CT scan show a lytic lesion in the left iliac bone with sclerotic margin and extension to soft tissue (Fig.1) To determine the histopathology of the mass and give a therapeutic design, an open biopsy was done. The specimen sent to the pathology ward and H\&E stained slides showed polymorphic inflammatory cell infiltration. Inflammatory infiltration was composed of reactive lymphocytes, eosinophils and plasma cells. In this reactive cellular background, scattered multilobated and mononuclear Reed-Stenberg cells and lacunar cells were detected. Immunohistochemistry was confirmatory; the large atypical cells were positive for CD15, CD30 and negative for CD20 (Fig. 2 and 3). The histopathology was consistent with a diagnosis of $\mathrm{CHL}$. CT of the neck, chest, and abdomen revealed no evidence of the cervical, mediastinal, or retroperitoneal lymph nodes involvement. After consulting with hematologists a treatment protocol of ABVD ICE chemotherapy was performed 
for this patient. Then he refered to radiotherapy center.

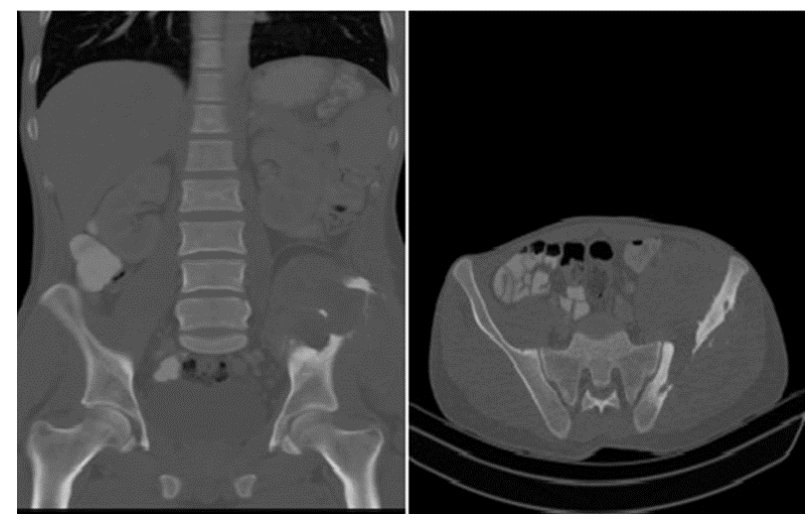

Figure 1: A Coronal and axial pelvic CT scan show a lytic lesion in the left iliac bone with sclerotic margin and extension to the soft tissue.

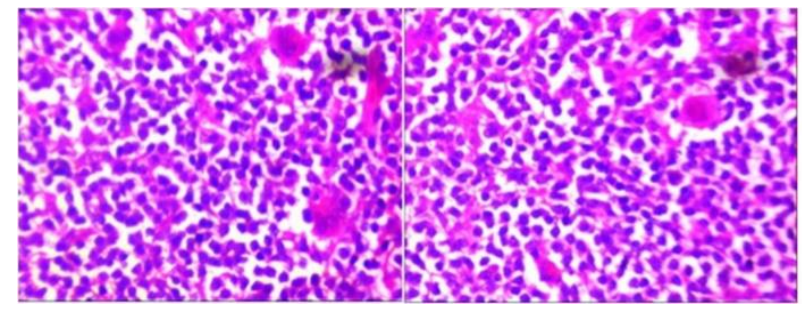

Figure 2: Sections show among reactive cellular background, scattered multilobated Reed-Stenberg cells and lacunar cells are detected (H\&E stain X 40).

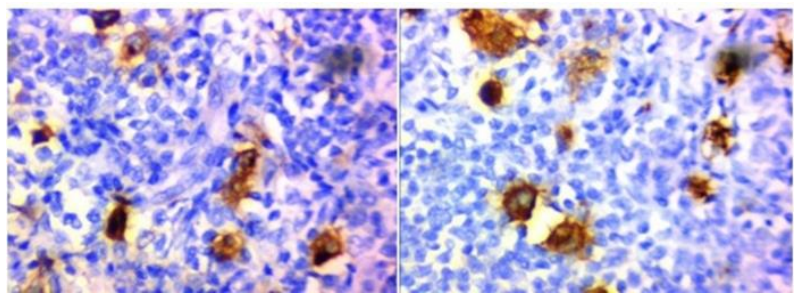

Figure 3: Neoplastic cells are positive for CD15 (left panel) andCD 30(right panel) (IHC stain X40).

\section{DISCUSSION}

As a matter of fact, $\mathrm{HL}$ involves the lymph nodes at one or more anatomical location. Bony entanglement in $\mathrm{HL}$ occurs through hematogenous spread or direct spread from the adjacent involved lymph node. Primary IDHL has been reported in $4-13 \%$ of patients ${ }^{[7]}$. Literature review showed primary pelvic $\mathrm{HL}$ is limited to adults ${ }^{[4,8]}$. Our patient was a 31 year old man. It is claimed that primary bony $\mathrm{HL}$ most frequently involve spine or long bone, and pelvic involvement is rare ${ }^{[2,4]}$. The present patient revealed pelvic bone involvement as a presenting symptom. Histologically, $\mathrm{CHL}$ has common features with other CD30 positive neoplasms such as primary mediastinal large Bcell lymphoma (PMBCL) and gray zone lymphoma. Hoeller et al showed that BOB.1, CD79a, and cyclin E are useful immunohistochemical markers that can assist recognizing $\mathrm{CHL}$ from PMBCL [9]. In addition, primary bony $\mathrm{HL}$ may be one differential diagnosis of other osseous neoplasms. For example, the primary diagnosis of Hodgkin lymphoma was malignant fibrous histiocytoma in one study [10]. The clinical presentation and imaging findings can be similar to other pelvic sarcomas such as chondrosarcoma, Ewing sarcoma, osteosarcoma sarcoma, and malignant fibrous histiocytoma [11]. Again histopathologic and IHC findings are crucial. In addition, treatment modality has clear distinction between $\mathrm{CHL}$ and other pelvic sarcomas [12]. Several specifications of IDHL compared with supradiaphragmatic $\mathrm{HL}$ have been explained. These include the older age, males' predominance and more frequent unflavored histology. On the other hand, Cédric Rossi, Morgane Mounier et al. suggest that the prognosis in PET-CT-staged IDHL may be similar to that in SDHL [13]. Some studies showed that patients with primary bony $\mathrm{HL}$ treated with combinedmodality therapy were found to have a longer survival than patients who underwent single modality $[14,15]$. Chemotherapy is yet the principle treatment choice for bony $\mathrm{HL}$ patient. Surgical approach is required just when there is a high risk of pathological fracture.

\section{CONCLUSION}

$\mathrm{CHL}$ at first presenting as pelvic involvement is actually scarce. The correct diagnosis should be made by tough histological and clinical manifestations.

\section{REFERENCES}

1. Ma J, Wang $\mathrm{Y}$, Zhao $\mathrm{H}$, Liu S, Li Q, Lin L, et al. Clinical characteristics of 26 patients with primary extranodal Hodgkin lymphoma. Int J Clin Exp Pathol. 2014; 7:5045-50.

2. Binesh F, Mirjalili MR, Akhavan A, Navabii H. Primary bony Hodgkin's lymphoma. BMJ Case Rep. 2012; 2012.

3. Gandhi JS, Mehta A, Sharma A, Kamboj M. Primary Hodgkin lymphoma of the ileum. J Cancer Res Ther. 2010; 6 (3):342-343.

4. Ha-ou-nou FZ, Benjilali L, Essaadouni L. Sacral pain as the initial symptom in primary Hodgkin's lymphoma of bone. J Cancer Res Ther. 2013; 9:511-3.

5. Biswas A, Puri T, Goyal S, Haresh KP, Gupta R, Julka PK, et al. Osseous Hodgkin's lymphoma-review of literature and report of an unusual case presenting as a large ulcerofungating sternal mass. Bone 2008; 43(3):636-640.

6. Vassilakopoulos TP, Angelopoulou MK, Siakantaris MP, Konstantinou N, Symeonidis A, Karmiris $\mathrm{T}$, et al. Hellenic Cooperative Lymphoma Group Pure infradiaphragmatic Hodgkin's lymphoma. Clinical features, prognostic factor and comparison with supradiaphragmatic disease. Haematologica. 2006; 91:32-39.

7. Darabi K, Sieber M, Chaitowitz M, Braitman LE, Tester W, DiehI V. Infradiaphragmatic versus supradiaphragmatic Hodgkin lymphoma: A retrospective review of 1,114 patients. Leuk Lymphoma. 2005; 46:1715-1720.

8. Kiran G, Canaz E, Sayar H, Serin S, Balakan O. Primary extranodal Hodgkin's lymphoma presenting with a single pelvic mass. J South Asian Fed Obstet Gynaecol. 2014; 6:183-6.

9. Hoeller S, Zihler D, Zlobec I, Obermann EC, Pileri SA, Dirnhofer S, et al. BOB.1, CD79a and cyclin E are the most appropriate markers to discriminate classical Hodgkin's lymphoma from primary mediastinal large B-cell lymphoma. Histopathology. 2010; 56:217228.

10. Ozdemirli M, Mankin HJ, Aisenberg AC, Harris NL. Hodgkin's disease presenting as a solitary bone tumor. A report of four cases and review of the literature. Cancer. 1996; 77:79-88.

11. Park SK, Lee IS, Cho KH, Lee YH, Yi JH, Choi KU. Osteosarcoma of pelvic bones: imaging features. Clin Imaging. 2017; 41:59-64.

12. Outani $\mathrm{H}$, Hamada $\mathrm{K}$, Imura $\mathrm{Y}$, Oshima $\mathrm{K}$, Sotobori $\mathrm{T}$, Demizu $\mathrm{Y}$, et al. Comparison of clinical and functional outcome between surgical treatment and carbon ion radiotherapy for pelvic chondrosarcoma. Int J Clin Oncol. 2016; 21:186-93.

13. Rossi C, Mounier M, Brice P, Safar V, Virelizier EN, Rey $P$, et al. Infradiaphragmatic Hodgkin lymphoma: a large series of patients staged with PET-CT. Oncotarget. 2017; 8(49):85110-85119.

14. Beal K, Allen L, Yahalom J. Primary bone lymphoma: treatment results and prognostic factors with long-term follow-up of 82 patients. Cancer 2006; 106:2652-6.

15. Tao R, Allen PK, Rodriguez A, Shihadeh F, Pinnix CC, Arzu I, et al. Benefit of consolidative radiation therapy for primary bone diffuse large B-cell lymphoma. Int J Radiat Oncol Biol Phys. 2015; 92(1):122-129. 